\title{
Recomendación clínica: evaluación preoperatoria
}

\section{Clinical guidelines: preoperative evaluation}

Ricardo Fuentes H. ${ }^{2}$, Claudio Nazar J. ${ }^{1}$, Patricio Vega G. ${ }^{2}$, Camila Stuardo M. ${ }^{3}$, Ángela Parra P. ${ }^{3}$, Waldo Merino U. ${ }^{4}$

\section{Abreviaturas}

SACH: Sociedad Chilena de Anestesiología.

GPC: Guía práctica clínica.

RC: Recomendación clínica.

MINSAL: Ministerio de Salud, Chile.

RCs: Recomendaciones clínicas.

GRADE: "The Grading of Recommendations Assessment, Development and Evaluation"

ECG: Electrocardiograma.

\section{Definición de términos}

1. Anestesia general: Estado reversible de pérdida de conciencia inducida por fármacos.

2. Anestesia regional: Conjunto de variadas técnicas anestésicas que permiten la inducción de insensibilidad en una zona del cuerpo, de forma controlada y reversible.

3. Evaluación ASA: Sistema de clasificación que utiliza la Sociedad Americana de Anestesiólogos (ASA).

1. Evaluación preanestésica: Proceso de evaluación y estudio que precede a la administración de anestesia, tanto para procedimientos quirúrgicos como no quirúrgicos.
2. Perioperatorio: Corresponde al período que se extiende desde que se indica la realización o necesidad de cirugía, hasta el momento en que el paciente es dado de alta.

3. Sedación: Estado farmacológico de depresión variable del estado de conciencia, cuya intención es facilitar el correcto desarrollo de procedimientos diagnósticos o terapéuticos, a la vez que proporciona comodidad y cooperación del paciente.

4. Unidad de Cuidados Posanestésicos: Sala destinada a proveer cuidados postanestésicos inmediatos de pacientes que han sido sometidos a cirugías o procedimientos diagnósticos o terapéuticos bajo anestesia, hasta que se alcancen criterios de alta predefinidos.

\section{Declaración de conflicto de interés}

Ricardo Fuentes Henríquez declara no tener conflicto de interés.

Claudio Nazar Jara declara no tener conflicto de interés.

Patricio Vega Guíñez declara no tener conflicto de interés.

Ángela Parra Palma declara no tener conflicto de interés.

Camila Stuardo Moraga declara no tener conflicto de interés.

\footnotetext{
Profesor Asociado, División de Anestesiología, Pontificia Universidad Católica de Chile. Instructor Asociado, División de Anestesiología, Pontificia Universidad Católica de Chile.

Residente de Anestesiología, Pontificia Universidad Católica de Chile.

Profesor Asistente, Departamento de Cirugía, Traumatología y Anestesia, Universidad de La Frontera.
}

Fecha de ingreso: 10 de enero de 2018

Fecha de aceptación: 05 de marzo de 2019

\section{ORCID}

https://orcid.org/0000-0002-4956-8444

Correspondencia:

Dr. Waldo Merino

Email: wamerino@gmail.com 


\begin{tabular}{llc}
\multicolumn{1}{c}{ Clasificación ASA } & \multicolumn{1}{c}{ Descripción } & Mortalidad perioperatoria \\
\hline I & Paciente sano, sin comorbilidad & $0-0,3 \%$ \\
II & Enfermedad sistémica leve a moderada, sin limitación funcional & $0,3-1,4 \%$ \\
III & Enfermedad sistémica moderada a severa, con limitación funcional & $1,8-5,4 \%$ \\
IV & Enfermedad sistémica severa, con riesgo vital constante & $7,8-25,9 \%$ \\
V & Paciente moribundo, con pocas probabilidades de sobrevivir las & $9,4-57,8 \%$ \\
& próximas 24 horas con o sin cirugía & $100 \%$ \\
\hline
\end{tabular}

Riesgo quirúrgico estimado de acuerdo al tipo de cirugía o intervención - (GUíA ESC/ESA 2014) -

\begin{tabular}{|c|c|}
\hline Riesgo & $\begin{array}{l}\text { Riesgo cardiovascular del procedimiento a } 30 \text { días de muerte o } \\
\text { infarto al miocardio propios de la cirugía e independiente de las } \\
\text { características del paciente[30] }\end{array}$ \\
\hline Riesgo alto (> 5\%) & $\begin{array}{l}\text { - Cirugía aórtica y vascular mayor } \\
\text { - Revascularización abierta de extremidad inferior, amputación o } \\
\text { tromboembolectomía } \\
\text { - Cirugía duodeno-pancreática } \\
\text { - Resección hepática, cirugías del ducto biliar } \\
\text { - Esofagectomía } \\
\text { - Reparación de víscera perforada } \\
\text { - Resección adrenal } \\
\text { - Cistectomía total } \\
\text { - Neumonectomía } \\
\text { - Trasplante hepático o pulmonar }\end{array}$ \\
\hline Riesgo intermedio (1-5\%) & $\begin{array}{l}\text { - Cirugías intraperitoneales: esplenectomía, reparación de hernia } \\
\text { hiatal, colecistectomía } \\
\text { - Cirugía carotída sintomática (endarterectomía e instalación } \\
\text { de stent) } \\
\text { - Angioplastía arterial periférica } \\
\text { - Reparación de aneurisma endovascular } \\
\text { - Cirugía de cabeza y cuello } \\
\text { - Cirugía neurológica u ortopédica mayor (ej: cadera y cirugía } \\
\text { de columna) } \\
\text { - Cirugía urológica o ginecológica mayor } \\
\text { - Trasplante renal } \\
\text { - Cirugía intratorácica }\end{array}$ \\
\hline Riesgo bajo $(<1 \%)$ & $\begin{array}{l}\text { - Cirugía superficial } \\
\text { - Cirugía de mamas } \\
\text { - Procedimientos dentales } \\
\text { - Cirugía tiroídea } \\
\text { - Cirugía ocular } \\
\text { - Cirugías reconstructivas } \\
\text { - Cirugías de carótida asintomática (endarterectomía e instalación } \\
\text { de stent) } \\
\text { - Cirugía ginecológica menor } \\
\text { - Cirugía ortopédica menor (menisectomía) } \\
\text { - Cirugía urológica menor (resección transureteral de la próstata) }\end{array}$ \\
\hline
\end{tabular}


Waldo Merino Urrutia declara no tener conflicto de interés.

\section{Desarrollo de la RC}

\subsection{Introducción}

La evaluación preanestésica consiste en la obtención de datos clínicos desde múltiples fuentes de información, como: información por otros médicos tratantes, la entrevista directa al paciente o sus representantes; un examen físico orientado a la intervención anestesiológica; y revisión de otras fuentes si las hubiere: ficha clínica, exámenes de laboratorio, otros documentos relevantes. Esta información es utilizada para organizar y planificar recursos en torno al acto anestésico, optimizando las condiciones y recursos disponibles o necesarios para reducir los riesgos asociados desde el tiempo de la evaluación hasta el momento del alta médica.

La evaluación preanestésica de un paciente que será sometido a un procedimiento quirúrgico o no quirúrgico es un pilar fundamental del cuidado anestésico, y cuando es realizada correctamente puede mejorar los resultados de los pacientes y su satisfacción, además de reducir los costos. Estas mejoras observadas en las últimas décadas han permitido la disminución de la mortalidad global en el perioperatorio. La evaluación preoperatoria está cambiando desde un tipo de evaluación "de rutina" a una evaluación de riesgo más individualizada. Este enfoque personalizado no sólo proporciona una mejor información sobre cada paciente, sino que también puede reducir significativamente los costos.

Actualmente siguen siendo escasos los estudios de buena calidad disponibles sobre el tema, y la mayor parte de las recomendaciones y guías clínicas internacionales se basan en opiniones de experto, las que posteriormente son adaptadas a los sistemas de salud de cada país.

\subsection{Objetivos}

La siguiente recomendación clínica tiene como propósito evaluar y dar a conocer la evidencia actualmente disponible sobre los efectos beneficiosos de la evaluación preanestésica y establecer la necesidad y condiciones para realizarla en nuestro quehacer clínico habitual.

Esta recomendación clínica servirá como base de referencia y apoyo de las conductas definidas y acordes a una correcta práctica profesional con respecto a la evaluación preanestésica, realizada por especia- listas o residentes en su periodo de formación y para conocimiento de los usuarios y adecuado estudio preoperatorio. Pretende ser utilizada como método de screening en la pesquisa de pacientes de riesgo y entregar herramientas para el uso racional de exámenes de laboratorio.

\subsubsection{Escenarios clínicos a los que se refiere la RC y condiciones de aplicación.} da:

La siguiente recomendación aplica para ser utiliza-

- Por anestesiólogos o aquellos que están bajo su supervisión directa.

- En pacientes sometidos a procedimientos electivos con anestesia general, anestesia regional o sedación moderada y profunda y/o monitorización, en pabellón o fuera de pabellón.

\subsubsection{Eventuales situaciones en las que no es aplicable la RC.}

Esta recomendación clínica no es aplicable a pacientes sometidos a procedimientos anestésicos realizados en situaciones de emergencia. Tampoco se aplica a pacientes sometidos a cirugía cardiaca o procedimientos dentales o sedaciones por operadores no especializados.

Esta recomendación no pretende establecer los procesos de evaluación de riesgo cardiovascular, respiratorio, entre otros, sino señalar las conductas recomendadas como método de screening en la pesquisa de pacientes de riesgo.

\subsection{Métodos}

Se realizó una búsqueda en las bases de datos MEDLINE/PUBMED, COCHRANE DATABASE OF SYSTEMATIC REVIEWS y LILACS. La búsqueda incluyó estudios y guías clínicas publicados entre los años 2007 y 2017, incluyendo aquellos publicados en inglés y español. Los términos usados en la búsqueda fueron: "preoperative assessment anesthesia" OR "preoperative evaluation" $O R$ "preoperative assessment" $O R$ "preanesthetic assessment" OR "preanesthetic evaluation" OR "preprocedural evaluation". La búsqueda desde Medline tuvo como resultado 1.054 estudios. Las bases Cochrane y Lilacs no agregaron nuevos artículos a los ya encontrados en Medline. De los 1.054 artículos, se preseleccionaron 110 artículos a través de la lectura de títulos, abstract o, en caso de ser necesario, el artículo completo. Posteriormente, 3 anestesiólogos conocedores del tema seleccionaron un total de 27 artículos[1]-[28], los que fueron utilizados en la confección de estas recomendaciones. 
Además de lo descrito en el párrafo anterior, se realizó una búsqueda dirigida de guías clínicas o recomendaciones de sociedades anestésicas reconocidas mundialmente y estamentos similares, tales como, Evaluación Preoperatoria del Paciente Sometido a Cirugía no Cardiaca de la Sociedad Europea de Anestesiología (ESA), Guías de Evaluación Preanestésica del Colegio de Anestesistas de Australia y Nueva Zelanda (ANZCA), Guías Prácticas de la Sociedad Americana de Anestesiología (ASA) y la Recomendación sobre Exámenes Preoperatorios de Rutina del Instituto Nacional de Salud y Excelencia Clínica del Reino Unido (NICE)[29]-[33].

Otra fuente de artículos a considerar fueron las referencias bibliográficas de los artículos seleccionados y descritos previamente, desde donde se seleccionaron 10 artículos adicionales[34]-[44]. Finalmente, se consideró el artículo previo sobre el tema publicado en la Revista Chilena de Anestesiología en agosto de 2007[45].

\subsubsection{Efectividad de las intervenciones.}

En la actualidad existen escasos estudios y revisiones sistemáticas sobre el tema. La mayor parte de las recomendaciones actuales se basan en guías clínicas de sociedades reconocidas internacionalmente, y que en su mayoría incluyen recomendaciones de expertos. Posteriormente, estas recomendaciones son adaptadas a la realidad local de los países.

\subsubsection{Efectos adversos de las intervenciones.}

La evaluación preoperatoria por sí misma no genera efectos adversos. Sin embargo, esta puede incluir intervenciones como la toma de exámenes, que no están exentas de causarlos. Los exámenes de laboratorio son útiles en guiar decisiones clínicas en el perioperatorio y su solicitud debe estar basada en el criterio clínico y evidencia cuando esta exista.

\subsubsection{Condiciones de calidad de las interven- ciones.}

La evaluación preanestésica de un paciente que será o no sometido a una cirugía debe ser realizada en un ambiente protegido para su pudor y seguridad. El examen físico es una parte relevante de la evaluación, por lo que ésta debe ser llevada a cabo con el paciente presente. El lugar en que se realiza debe ser privado, debido a que la entrevista contiene preguntas sensibles. Si no se cuenta con una consulta privada para realizarla, recomendamos la unidad de cuidados preanestésicos como una opción. Además, el lugar debe contar con los implementos necesarios que permitan examinar y tomar los signos vitales del paciente.

\subsubsection{Métodos de consenso utilizados para la formulación de recomendaciones.}

La participación de los autores se logró mediante la realización de reuniones semanales vía videoconferencia, en las cuales inicialmente se tomaron decisiones respecto a los lineamientos generales del informe, cuyos avances eran, además, revisados semanalmente. En la formulación de la RC se establecieron controversias que pudieron encontrarse en la apreciación de la evidencia disponible con respecto a:

- La validez de los estudios para el contexto chileno.

- La relevancia clínica de los resultados.

- El balance entre los desenlaces favorables y desfavorables.

- Las condiciones de aplicabilidad o el impacto esperado de las recomendaciones.

- Incorporación de nuevos artículos en la búsqueda de evidencia o bibliografía.

- Las evidencias encontradas resultaran contradictorias o las condiciones locales no hicieran aplicable la recomendación.

Los conflictos suscitados durante la realización de las presentes recomendaciones se resolvieron en reuniones separadas con todos los miembros del grupo de trabajo presentes, donde se discutió el conflicto y se planteó la disponibilidad de información básica para la toma de estas decisiones, resolviendo finalmente según lo adoptado por la mayoría, con especial peso otorgado a la opinión de los expertos participantes. Así, para resolver estas diferencias, se aplicaron métodos de consenso, que aseguraron:

- Representatividad: Todos los autores o al menos tres de ellos y eventualmente el miembro del comité de RCs participó de su resolución.

- Todos los miembros del grupo a cargo de la elaboración del documento dispusieron del mismo nivel de información básica para la toma de decisiones.

- Las diferencias se resolvieron a través de votación por mayoría simple, cuando no se alcanzó consenso o para la aprobación de este.

\subsubsection{Grados de evidencia y niveles de recomen- dación utilizados en la RCs.}

En la elaboración de esta RC se efectuó de acuerdo con los lineamientos entregados por la SACH a través del documento "Orientaciones para la Elaboración y Actualización de Recomendaciones Clínicas de la Sociedad de Anestesiología de Chile".

Para la evaluación y redacción de las recomendaciones se utilizó el sistema GRADE recomendado por el MINSAL. Sin embargo, es necesario mencionar lo 
establecido por el MINSAL que “... no todas las preguntas relevantes para una guía de práctica clínica pueden, ni deben, responderse con evidencia científica. Cuando las características de una intervención hacen que no sea posible o éticamente válido realizar estudios respecto de su efectividad, y se considera en consenso que dicha intervención refleja un estándar mínimo de atención, el panel puede emitir una recomendación de buena práctica".

\section{Recomendaciones}

\subsection{Momento de la evaluación}

\subsubsection{Este grupo de trabajo considera imprescin-} dible recomendar:

1. Realizar la evaluación preoperatoria con tiempo suficiente antes del procedimiento programado, para permitir la implementación de cualquier intervención preoperatoria aconsejable dirigida a mejorar el resultado del paciente[32].

2. En caso de procedimientos de alta invasividad o en pacientes con enfermedades sistémicas severas, recomendamos realizar la evaluación preanestésica el día antes de la cirugía[31].

3. En procedimientos de baja o moderada invasividad y con patologías sistémicas de bajo riesgo, la evaluación preanestésica se puede realizar el mismo día de la cirugía[31].

\subsubsection{La institución hospitalaria debe proporcio-} nar al equipo de anestesiología en forma oportuna la información necesaria para llevar a cabo una adecuada y completa evaluación preanestésica (incluyendo información sobre los casos programados, acceso completo y expedito a toda la ficha clínica, recintos de buena calidad para hacer un examen físico y monitores mínimos para medir signos vitales). La información de aquellos pacientes que serán intervenidos en forma electiva y que comúnmente están contenidos en una ficha clínica electrónica o convencional, debe incluir al menos antecedentes médicos del paciente, procedimiento quirúrgico propuesto y resultados de exámenes de laboratorio necesarios para una correcta evaluación perioperatoria[31].

\subsubsection{La evaluación en los términos antes seña-} lados, debe ser realizada por un médico anestesiólogo o por quien se encuentre bajo su supervisión directa[32],[52],[53],[54].

La calidad de la evidencia es alta. La recomendación fuerte.
5.1.4 Recomendamos considerar la entrega de información al paciente respecto al plan anestésico formulado, los procedimientos que éste involucra y los riesgos asociados[33]. Debido a la frecuencia se debe hacer especial hincapié en la posibilidad de pérdida de piezas dentales al manipular la vía aérea, especialmente en pacientes que poseen dentadura en mal estado.

Comentario: El equipo de trabajo considera escasa la bibliografía que sustenta esta recomendación. Sin embargo, en condición de expertos, recomendamos fuertemente su aplicación, con la finalidad de que el paciente conozca el plan anestésico y los riesgos implicados.

\subsection{Historia y examen físico}

5.2.1 Recomendamos realizar la evaluación del paciente en un ambiente protegido, garantizando privacidad tanto en la entrevista como en el examen físico[33].

5.2.2 Este equipo de trabajo a determinado recomendar que la evaluación preanestésica debe contener una revisión del historial médico, entrevista con el paciente y examen físico[31].

5.2.3 Este grupo de trabajo considera importante recomendar que[33]:

1. En la evaluación preanestésica, la persona encargada de efectuarla, porte de manera visible una tarjeta de identificación o documento de características similares (tal como indica la ley vigente 20.584).

2. En la evaluación preanestésica se debe confirmar y consignar la identidad del paciente (incluyendo nombre y número de cédula de identidad) y el procedimiento propuesto, incluyendo el sitio anatómico a intervenir.

5.2.4 La historia médica debe contener una descripción de las patologías actuales y sus tratamientos, incluyendo medicamentos y terapias alternativas, antecedente de cirugías o procedimientos previos, la revisión o interrogación sobre procedimientos anestésicos previos y sus eventuales complicaciones y el resultado de exámenes relevantes[31],[33],[55]-[63].

La calidad de la evidencia es moderada. La recomendación fuerte.

5.2.5 El examen físico debe incluir, como mínimo, una evaluación de la vía aérea, un examen cardiopulmonar y un registro de los signos vitales[31]. 
5.2.6 Recomendamos confeccionar una hoja de registro de la evaluación preanestésica la cual puede ser completada por el anestesista en forma de papel o electrónica de acuerdo a la situación de cada institución hospitalaria. Este registro debiera ser un cuestionario estandarizado y en su diseño se debe considerar al menos los siguientes campos: identificación del paciente, fecha de evaluación, diagnóstico y procedimiento a realizar, anamnesis remota (incluyendo alergias, hábitos, fármacos, antecedentes médicos, quirúrgicos y anestésicos), revisión por sistemas, período de ayuno, examen físico dirigido, resultados de exámenes de laboratorio relevantes, evaluación ASA y plan anestésico sugerido al paciente.

5.2.7 Como parte del papel de la anestesiología en el cuidado de la salud, recomendamos que la evaluación preanestésica sea una instancia para fomentar y educar a los pacientes en relación a cambios en el estilo de vida o a la modificación de conductas de riesgo, tales como tabaquismo, alcoholismo, sedentarismo, entre otros[33].

\subsection{Exámenes}

\subsubsection{Hemograma}

5.3.1.1 No recomendamos la obtención de un hematocrito de forma rutinaria. Más bien, una historia clínica individual y un examen físico deben guiar su obtención[3],[31].

5.3.1.2 Recomendamos solicitar hematocrito en pacientes seleccionados en base a condiciones que aumentan la probabilidad de anemia: historia de sangrado, enfermedad inflamatoria crónica, enfermedad renal crónica, enfermedad hepática crónica, signos clínicos o síntomas de anemia u otros trastornos hematológicos, quimioterapia, radioterapia, extremos de edad o procedimientos en los que se prevé una pérdida significativa de sangre ( $>500 \mathrm{ml}$ en adultos $\mathrm{y}$ $7 \mathrm{ml} / \mathrm{kg}$ en niños)[23],[31].

5.3.1.3 Este grupo de trabajo considera importante recomendar que[29]:

1. En pacientes que serán sometidos a cirugías de bajo riesgo, no recomendamos la solicitud de un hemograma de forma rutinaria.

2. En pacientes que serán sometidos a cirugías de riesgo intermedio, recomendamos su solicitud en pacientes ASA 3 o 4 con enfermedad cardiovascular o enfermedad renal.
3. Se recomienda su solicitud en todo paciente que será sometido a cirugías de alto riesgo quirúrgico.

5.3.2 Función renal y electrolitos[7],[29],[31].

5.3.2.1 No recomendamos el estudio de la función renal de forma rutinaria en el preoperatorio.

5.3.2.2 La solicitud de una creatinina plasmática se justifica en pacientes con antecedentes de enfermedad renal crónica.

5.3.2.3 Recomendamos evaluar la función renal en aquellos pacientes que[29],[64]:

- Tengan antecedentes de diabetes mellitus, arteriosclerosis, enfermedad hepática, insuficiencia cardíaca, enfermedad coronaria.

- En pacientes sépticos o en contexto de hipovolemia cuando sea posible.

- Mayores de 65 años.

- En aquellos pacientes en que se prevea el uso potencial de drogas nefrotóxicas en el perioperatorio[29],[64].

La calidad de la evidencia es muy baja. La recomendación fuerte.

5.3.2.4 Sugerimos la determinación de electrolitos en pacientes que tengan una historia que aumente la probabilidad de encontrar alteraciones[44], tales como antecedentes de enfermedad renal crónica conocida, enfermedad hepática, insuficiencia cardíaca congestiva o historia de uso de medicamentos que afecten los niveles de electrolitos, tales como diuréticos, inhibidores de la enzima convertidora de la angiotensina (iECA) o bloqueadores de los receptores de la angiotensina (ARA II)[7],[44].

\subsubsection{Glicemia}

5.3.3.1 Sugerimos la evaluación preoperatoria de los niveles de glucosa plasmática cuando exista sospecha clínica de alteraciones en el metabolismo de la glu$\operatorname{cosa}[3]$.

5.3.3.2 Recomendamos la obtención de niveles de glicemia en el preoperatorio en pacientes con mayor incidencia de infección de herida operatoria y complicaciones cardiovasculares en relación a la hiperglicemia, tales como: pacientes sometidos a cirugía vascular, cirugía ortopédica y cirugía de columna vertebral (sin diagnóstico de diabetes conocido)[3],[65]-[74]. La calidad de la evidencia es moderada. La recomendación fuerte. 


\subsubsection{Pruebas de coagulación}

5.3.4.1 Recomendamos no realizar estudio de pruebas de coagulación de forma rutinaria en el preoperatorio[3],[75].

La calidad de la evidencia es moderada. La recomendación fuerte.

5.3.4.2 Recomendamos solicitar recuento de plaquetas, tiempo de protrombina y tiempo parcial de tromboplastina activada en pacientes con comorbilidades asociadas a alteración de la hemostasia (enfermedad hepática, enfermedad renal), usuarios de anticoagulantes, alteraciones de la hematopoyesis y en aquellos cuyos antecedentes o hallazgos en la historia o examen físico sugieran un trastorno de la coagulación subyacente (ej: historia de sangrado excesivo, antecedentes familiares de coagulopatía hereditaria conocida)[3],[4],[31],[32],[44].

\subsubsection{Test de embarazo}

5.3.5.1 Recomendamos la realización de un test de embarazo a todas aquellas mujeres en edad fértil en las que exista la duda sobre si pudiera o no estar embarazada, y en aquellas en las cuales un embarazo podría resultar en cambios en el manejo, tanto anestésico como quirúrgico[3],[29].

\subsubsection{Electrocardiograma (ECG)}

5.3.6.1 Recomendamos la realización de un ECG de reposo de 12 derivadas en pacientes con enfermedad coronaria conocida, enfermedades pulmonares, arritmias, enfermedad arterial periférica, enfermedad cerebrovascular y otras enfermedades cardíacas estructurales, como también en pacientes diabéticos insulino-requirientes o aquellos con enfermedad renal (creatinina $>2 \mathrm{mg} / \mathrm{dl}$ o clearence de creatinina $<60$ $\mathrm{ml} / \mathrm{min} / 1,73 \mathrm{~m}^{2}$ ), así como en aquellos con signos o síntomas cardiovasculares activos y que serán sometidos a cirugías de riesgo cardíaco elevado (> 1\%) [3],[30],[31],[38].

La calidad de la evidencia es moderada. La recomendación fuerte.

5.3.6.2 No recomendamos la realización de un ECG de reposo en pacientes sin factores de riesgo que serán sometidos a cirugías de bajo riesgo[30].

5.3.6.3 Sugerimos la realización de un ECG de reposo en pacientes con factores de riesgo, incluso cuando serán sometidos a cirugías de bajo riesgo[30].
5.3.6.4 En pacientes mayores de 65 años, sin factores de riesgo, que serán sometidos a cirugías de riesgo intermedio y alto[30].

La calidad de la evidencia es baja. La recomendación fuerte.

5.3.6.5 El equipo de trabajo considera que el ECG tiene una validez de 6 meses, siempre que no se produzcan cambios clínicos importantes en la condición del paciente durante este intervalo de tiempo[15].

\subsubsection{Ecocardiograma}

5.3.7.1 Este grupo de trabajo recomienda solicitar un ecocardiograma en pacientes con signos o síntomas de insuficiencia cardiaca, como soplo cardíaco y síntomas cardíacos como disnea, síncope, dolor torácico[ 29].

\subsubsection{Radiografía de tórax}

5.3.8.1 Sugerimos considerar radiografía de tórax frente a pacientes con signos o síntomas de condiciones cardiopulmonares nuevas o inestables[42].

\subsubsection{Examen de orina}

5.3.9.1 Recomendamos solicitar exámenes de orina (cultivo y/o microscopía) si se sospecha infección urinaria y si la realización de este examen puede cambiar alguna decisión de la cirugía[29],[76].

5.3.9.2 Sugerimos solicitar urocultivo en pacientes que serán sometidos a procedimientos urológicos específicos o a la instalación de prótesis con material foráneo (prótesis de articulaciones, válvulas cardíacas) [44].

\section{Repercusiones económicas potencialmente re- levantes de la aplicación de la RC}

El proceso de tamizaje o screening mediante la evaluación preanestésica de los pacientes que serán sometidos a cirugías o procedimientos no quirúrgicos permite mejorar el estado del paciente previo a la realización del procedimiento, minimizando los costos y optimizando los resultados. Posibilita, además, la disminución en los retrasos o cancelaciones de procedimientos, asegurándose que las exploraciones complementarias se realicen, evitando así la realización rutinaria de pruebas o interconsultas innecesarias que suponen una sobrecarga económica y estructural del sistema de salud. 
Se ha estimado que entre un 60 a un $75 \%$ de los exámenes preoperatorios solicitados son clínicamente innecesarios (https://www.ncbi.nlm.nih.gov/pmc/articles/PMC3016569/). La realización indiscriminada de exámenes puede aumentar el riesgo de daño iatrogénico derivado de exámenes o tratamientos innecesarios cuando se obtiene un resultado límite o un falso positivo. Un resultado falso positivo distrae al médico de detectar un problema clínicamente significativo y puede eventualmente dañar al paciente. Además, los exámenes innecesarios pueden causar un retraso o cancelación de cirugías planificadas. Es mejor no solicitar un examen innecesario, pues el riesgo médico legal es mayor por no seguir un resultado de un examen anormal, que por no solicitar un examen que no estaba indicado.

\section{Referencias}

1. Ministerio de Salud. División de Planificación y Presupuesto. Departamento Calidad de Prestadores. Instructivo: establece requisitos básicos para la elaboración de guías clínicas y protocolos del Ministerio de Salud. Santiago, Chile; 2000.

2. Auckley D, Bolden N. Preoperative screening and perioperative care of the patient with sleepdisordered breathing. Curr Opin Pulm Med. 2012 Nov;18(6):58895. https://doi.org/10.1097/ MCP.0b013e3283589e6e PMID:22990655

3. Bock M, Fritsch G, Hepner DL. Preoperative Laboratory Testing. Anesthesiol Clin. 2016 Mar;34(1):43-58. https://doi.org/10.1016/j. anclin.2015.10.005 PMID:26927738

4. Boehm O, Baumgarten G, Hoeft A. Preoperative patient assessment: identifying patients at high risk. Best Pract Res Clin Anaesthesiol. 2016 Jun;30(2):131-43. https://doi. org/10.1016/j.bpa.2016.04.005 PMID:27396802

5. Chang B, Urman RD. Nonoperating Room Anesthesia: The Principles of Patient Assessment and Preparation. Anesthesiol Clin. 2016 Mar;34(1):22340. https://doi.org/10.1016/j. anclin.2015.10.017 PMID:26927750
6. Chow WB, Rosenthal RA, Merkow RP, Ko CY, Esnaola NF; American College of Surgeons National Surgical Quality Improvement Program; American Geriatrics Society. Optimal preoperative assessment of the geriatric surgical patient: a best practices guideline from the American College of Surgeons National Surgical Quality Improvement Program and the American Geriatrics Society. J Am Coll Surg. 2012 Oct;215(4):45366. https://doi.org/10.1016/j. jamcollsurg.2012.06.017 PMID:22917646

7. Cohn SL. Preoperative evaluation for noncardiac surgery. Ann Intern Med. 2016;165(11):ITC8294.

8. Cohn SL. Updated guidelines on cardiovascular evaluation before noncardiac surgery: a view from the trenches. Cleve Clin J Med. 2014 Dec;81(12):742-51. https:// doi.org/10.3949/ccjm.81a.14148 PMID:25452352

9. Conner B. Pulmonary function testing. Immunol Allergy Clin North Am. 1999;19(1):47-61. https://doi.org/10.1016/S08898561(05)70053-9.

10. Dewan SK, Zheng SB, Xia SJ. Preoperative geriatric assessment: comprehensive, multidisciplinary and proactive. Eur J Intern Med. 2012 Sep;23(6):487-94. https://doi. org/10.1016/j.ejim.2012.06.009 PMID:22863423
11. Dhatariya K, Levy $N$, Kilvert A, Watson B, Cousins D, Flanagan $D$, et al.; Joint British Diabetes Societies. NHS Diabetes guideline for the perioperative management of the adult patient with diabetes. Diabet Med. 2012 Apr;29(4):42033. https://doi.org/10.1111/ j.1464-5491.2012.03582.x PMID:22288687

12. Duncan D, Wijeysundera DN. Preoperative Cardiac Evaluation and Management of the Patient Undergoing Major Vascular Surgery. Int Anesthesiol Clin. 2016;54(2):132. https://doi.org/10.1097/ AIA.0000000000000091 PMID:26967800

13. Fleisher LA. Preoperative Assessment of the Patient with Cardiac Disease Undergoing Noncardiac Surgery. Anesthesiol Clin. 2016 Mar;34(1):5970. https://doi.org/10.1016/j. anclin.2015.10.006 PMID:26927739

14. Frost EAM. Preoperative evaluation. Seminars in Anesthesia, Perioperative Medicine and Pain [Internet]. Elsevier BV; 2005 Jun;24(2):80-8. Available from: http://dx.doi.org/10.1053/j. sane.2005.04.003.

15. Gooden CK, Frost EA. Preprocedural evaluation: considerations outside of the operating room. Curr Opin Anaesthesiol. 2015 Aug;28(4):441-5. https://doi.org/10.1097/ 
ACO.0000000000000217 PMID:26087266

16. Gupta A. Preoperative screening and risk assessment in the ambulatory surgery patient. Curr Opin Anaesthesiol. 2009 Dec;22(6):705-11. https://doi.org/10.1097/ ACO.0b013e3283301fb3 PMID:19633545

17. Hofer J, Chung E, Sweitzer BJ. Preanesthesia evaluation for ambulatory surgery: do we make a difference? Curr Opin Anaesthesiol. 2013 Dec;26(6):669-76. https://doi.org/10.1097/ AC0.0000000000000006 PMID:24184883

18. King MS. Preoperative evaluation. Am Fam Physician. 2000 Jul;62(2):387-96. PMID:10929701

19. Knittel JG, Wildes TS. Preoperative Assessment of Geriatric Patients. Anesthesiol Clin. 2016 Mar;34(1):171-83. https://doi.org/10.1016/j. anclin.2015.10.013 PMID:26927746

20. Miller JD, Richman DC. Preoperative Evaluation of Patients with Diabetes Mellitus. Anesthesiol Clin. 2016 Mar;34(1):15569. https://doi.org/10.1016/j. anclin.2015.10.008 PMID:26927745

21. Mittnacht AJ, Fanshawe M, Konstadt S. Anesthetic considerations in the patient with valvular heart disease undergoing noncardiac surgery. Semin Cardiothorac Vasc Anesth. 2008 Mar;12(1):33-59. https://doi. rg/10.1177/1089253208316442 PMID:18397904

22. Nørskov AK. Preoperative airway assessment - experience gained from a multicentre cluster randomised trial and the Danish Anaesthesia Database. Dan Med J. 2016 May;63(5):1-17. PMID:27127020

23. Richman DC. Ambulatory surgery: how much testing do we need? Anesthesiol

Clin. 2010 Jun;28(2):185-97. https://doi.org/10.1016/j. anclin.2010.03.001 PMID:20488389

24. Schiefermueller J, Myerson S, Handa Al. Preoperative assessment and perioperative management of cardiovascular risk. Angiology. 2013 Feb;64(2):146-50. https://doi. rg/10.1177/0003319712440874 PMID:22563034

25. Seet E, Chung F. Obstructive sleep apnea: preoperative assessment. Anesthesiol Clin. 2010 Jun;28(2):199-215. https://doi.org/10.1016/j. anclin.2010.02.002 PMID:20488390

26. Smith I, Kranke $P$, Murat I, Smith A, O'Sullivan G, Søreide E, et al, European Society of Anaesthesiology. Perioperative fasting in adults and children: guidelines from the European Society of Anaesthesiology. Eur J Anaesthesiol. 2011 Aug;28(8):55669. https://doi.org/10.1097/ EJA.0b013e3283495ba1 PMID:21712716

27. White PF, White LM, Monk T, Jakobsson J, Raeder J, Mulroy MF, et al. Perioperative care for the older outpatient undergoing ambulatory surgery. Anesth Analg. 2012 Jun;114(6):1190215. https://doi.org/10.1213/ ANE.0b013e31824f19b8 PMID:22467899

28. Zraier S, Haouache H, Dhonneur $\mathrm{G}$. Which preoperative respiratory evaluation? Ann Fr Anesth Reanim. 2014 Jul-Aug;33(7-8):453-6. https://doi.org/10.1016/j. annfar.2014.07.743 PMID:25168302

29. NICE. Routine preoperative tests for elective surgery. NICE Clinical Guidelines. 2016. Obtenido desde https://www.nice.org.uk/ guidance/ng45

30. Kristensen SD, Knuuti J, Saraste A, Anker S, Bøtker HE, Hert SD, et al.; Authors/Task Force Members. 2014 ESC/ESA Guidelines on non-cardiac surgery: cardiovascular assessment and management: The Joint Task Force on non-cardiac surgery: cardiovascular assessment and management of the European Society of Cardiology (ESC) and the European Society of Anaesthesiology (ESA). Eur Heart J. 2014 Sep;35(35):2383-431. https://doi.org/10.1093/eurheartj/ehu282 PMID:25086026

31. Apfelbaum JL, Connis RT, Nickinovich DG, Pasternak LR, Arens JF, Caplan RA, et al.; American Society of Anesthesiologists Task Force on Preanesthesia Evaluation. Practice advisory for preanesthesia evaluation: an updated report by the American Society of Anesthesiologists Task Force on Preanesthesia Evaluation. Anesthesiology. 2012 Mar;116(3):522-38. https://doi.org/10.1097/ ALN.0b013e31823c1067 PMID:22273990

32. De Hert $S$, Imberger $G$, Carlisle J, Diemunsch P, Fritsch G, Moppett I, et al.; Task Force on Preoperative Evaluation of the Adult Noncardiac Surgery Patient of the European Society of Anaesthesiology. Preoperative evaluation of the adult patient undergoing non-cardiac surgery: guidelines from the European Society of Anaesthesiology. Eur J Anaesthesiol. 2011 Oct;28(10):684722. https://doi.org/10.1097/ EJA.0b013e3283499e3b PMID:21885981

33. Consultation, P., \& Preparation, P. (2016). Ps07 2016, 1-8.

34. Barker $P$, Creasey PE, Dhatariya K, Levy N, Lipp A, Nathanson $\mathrm{MH}$, et al.; Membership of the Working Party. Peri-operative 
management of the surgical patient with diabetes 2015: association of Anaesthetists of Great Britain and Ireland. Anaesthesia. 2015 Dec;70(12):142740. https://doi.org/10.1111/ anae. 13233 PMID:26417892

35. Chung F, Yuan H, Yin L, Vairavanathan $S$, Wong DT. Elimination of preoperative testing in ambulatory surgery. Anesth Analg. 2009 Feb;108(2):46775. https://doi.org/10.1213/ ane.0b013e318176bc19 PMID:19151274

36. Devereaux PJ, Beattie WS, Choi PT, Badner NH, Guyatt GH, Villar JC, et al. How strong is the evidence for the use of perioperative beta blockers in non-cardiac surgery? Systematic review and meta-analysis of randomised controlled trials. BMJ. 2005 Aug;331(7512):31321. https://doi.org/10.1136/ bmj.38503.623646.8F PMID:15996966

37. Devereaux PJ, Yang $H$, Yusuf S, Guyatt G, Leslie K, Villar JC, et al.; POISE Study Group. Effects of extended-release metoprolol succinate in patients undergoing non-cardiac surgery (POISE trial): a randomised controlled trial. Lancet. 2008 May;371(9627):183947. https://doi.org/10.1016/ S0140-6736(08)60601-7 PMID:18479744

38. Fleisher LA, Fleischmann KE, Auerbach AD, Barnason SA, Beckman JA, Bozkurt B, et al. 2014 ACC/AHA guideline on perioperative cardiovascular evaluation and management of patients undergoing noncardiac surgery: executive summary: a report of the American College of Cardiology/American Heart Association Task Force on Practice Guidelines. Circulation. 2014 Dec;130(24):221545. https://doi.org/10.1161/

\section{CIR.0000000000000105 PMID:25085962}

39. Johansson $T$, Fritsch $G$, Flamm M, Hansbauer B, Bachofner N, Mann E, et al. Effectiveness of non-cardiac preoperative testing in non-cardiac elective surgery: a systematic review. Br J Anaesth. 2013 Jun;110(6):926-39. https:// doi.org/10.1093/bja/aet071 PMID:23578861

40. Juul $A B$, Wetterslev J, Gluud C, Kofoed-Enevoldsen $A$, Jensen $G$, Callesen T, et al.; DIPOM Trial Group. Effect of perioperative beta blockade in patients with diabetes undergoing major non-cardiac surgery: randomised placebo controlled, blinded multicentre trial. BMJ. 2006 Jun;332(7556):1482. https://doi. org/10.1136/bmj.332.7556.1482 PMID:16793810

41. Lindström D, Sadr Azodi O, Wladis A, Tønnesen $\mathrm{H}$, Linder $\mathrm{S}$, Nåsell $H$, et al. Effects of a perioperative smoking cessation intervention on postoperative complications: a randomized trial. Ann Surg. 2008 Nov;248(5):73945. https://doi.org/10.1097/ SLA.0b013e3181889d0d PMID:18948800

42. Smetana GW, Lawrence VA, Cornell JE. Annals of Internal Medicine Clinical Guidelines Preoperative Pulmonary Risk Stratification for Noncardiothoracic Surgery: Systematic Review for the American College of Physicians. 2006

43. Sweitzer BJ. Preoperative screening, evaluation, and optimization of the patient's medical status before outpatient surgery. Curr Opin Anaesthesiol. 2008 Dec;21(6):711-8. https://doi.org/10.1097/ ACO.0b013e3283126cf3 PMID:18997522

44. Feely MA, Collins CS, Daniels PR, Kebede EB, Jatoi A, Mauck KF. Preoperative testing before noncardiac surgery: guidelines and recommendations. Am Fam Physician. 2013 Mar;87(6):4148. PMID:23547574

45. Bustamante R. Recomendaciones Sobre Evaluación Preanestésica. Revista Chilena de Anestesia. 2007;230:200-30.

46. Manual Metodológico de Guías de Práctica Clínica. MINSAL. ${ }^{a}$ Edición: septiembre, 2014. Santiago de Chile.

47. El sistema GRADE: un cambio en la forma de evaluar la calidad de la evidencia y la fuerza de recomendaciones. Ignacio Neumann1,2,5,6,7, Tomás Pantoja1,3, Blanca Peñaloza1,3, Lorena Cifuentes1,4, Gabriel Rada1,2,6,7. Rev Med Chile. 2014;142:630-5. https://doi.org/10.4067/S003498872014000500012.

48. Kristensen SD, Knuuti J, Saraste A, Anker S, Bøtker HE, De Hert S, et al.; Authors/Task Force Members. 2014 ESC/ESA Guidelines on non-cardiac surgery: cardiovascular assessment and management: The Joint Task Force on non-cardiac surgery: cardiovascular assessment and management of the European Society of Cardiology (ESC) and the European Society of Anaesthesiology (ESA). Eur J Anaesthesiol. 2014 Oct;31(10):517-73. https://doi.org/10.1097/ EJA.0000000000000150 PMID:25127426

49. Recommendations for guidelines productions. A document for task forces members responsible for the production un updating of ESC Guidelines. Committe for Practice Guidelines of the European Society of Cardiology. https://www.escardio.org/ static_file/Escardio/Guidelines/ about/ESC_Guidelines_for_Guidelines_Update_2012_for_web. pdf

50. "Orientaciones para la elabora- 
ción y actualización de Recomendaciones Clínicas de la Sociedad de Anestesiología de Chile" Comité de Recomendaciones Clínicas SACH. 2017.

51. Bustamante R. Editorial: recomendaciones Clínicas. Rev Chil Anest. 2012;41:163-5.

52. Kinley H, Czoski-Murray C, George S, McCabe C, Primrose J, Reilly C, et al. Effectiveness of appropriately trained nurses in preoperative assessment: randomised controlled equivalence/ non-inferiority trial. BMJ. 2002 Dec;325(7376):1323. https://doi. org/10.1136/bmj.325.7376.1323 PMID:12468478

53. Rushforth $H$, Burge $D$, Mullee $M$, Jones S, McDonald H, Glasper EA. Nurse-led paediatric pre operative assessment: an equivalence study. Paediatr Nurs. 2006 Apr;18(3):23-9. PMID:16634381

54. Hilditch WG, Kopka A, Crawford JM, Asbury AJ. Interobserver reliability between a nurse and anaesthetist of tests used for predicting difficult tracheal intubation [REVISTA??]. Anaesthesia. 2004 Sep;59(9):881-4. https://doi.org/10.1111/ j.1365-2044.2004.03740.x PMID:15310351

55. Boersma E, Kertai MD, Schouten O, Bax JJ, Noordzij P, Steyerberg EW, et al. Perioperative cardiovascular mortality in noncardiac surgery: validation of the Lee cardiac risk index. Am J Med. 2005 Oct;118(10):113441. https://doi.org/10.1016/j. amjmed.2005.01.064 PMID:16194645

56. Canet J, Gallart L, Gomar C, Paluzie G, Vallès J, Castillo J, et al.; ARISCAT Group. Prediction of postoperative pulmonary complications in a populationbased surgical cohort. Anesthesiology. 2010 Dec;113(6):133850. https://doi.org/10.1097/ ALN.0b013e3181fc6e0a
PMID:21045639

57. Cohen MM, Duncan PG. Physical status score and trends in anesthetic complications. J Clin Epidemiol. 1988;41(1):83-90. https://doi.org/10.1016/08954356(88)90012-1 PMID:3335873

58. Correll DJ, Hepner DL, Chang C, Tsen L, Hevelone ND, Bader AM. Preoperative electrocardiograms: patient factors predictive of abnormalities. Anesthesiology. 2009 Jun;110(6):121722. https://doi.org/10.1097/ ALN.0b013e31819fb139 PMID:19417620

59. Cullen DJ, Apolone G, Greenfield S, Guadagnoli E, Cleary P. ASA Physical Status and age predict morbidity after three surgical procedures. Ann Surg. 1994 Jul;220(1):3-9. https:// doi.org/10.1097/00000658199407000-00002 PMID:8024356

60. Dripps RD, Lamont A, Eckenhoff JE. The role of anesthesia in surgical mortality. JAMA. 1961 Oct;178(3):261-6. https://doi.org/10.1001/ jama.1961.03040420001001 PMID:13887881

61. Dudley JC, Brandenburg JA, Hartley LH, Harris S, Lee TH. Last-minute preoperative cardiology consultations: epidemiology and impact. Am Heart J. 1996 Feb;131(2):2459. https://doi.org/10.1016/ S0002-8703(96)90348-8 PMID:8579015

62. Dzankic S, Pastor D, Gonzalez C, Leung JM. The prevalence and predictive value of abnormal preoperative laboratory tests in elderly surgical patients. Anesth Analg. 2001 Aug;93(2):301-8. PMID:11473849

63. Gibbs J, Cull W, Henderson W, Daley J, Hur K, Khuri SF. Preoperative serum albumin level as a predictor of operative mortality and morbidity: results from the National VA Surgical Risk Study. Arch Surg. 1999 Jan;134(1):3642. https://doi.org/10.1001/archsurg.134.1.36 PMID:9927128

64. Acute kidney Injury. Prevention, detection and management up to the point of renal replacement therapy. Clinical Guideline CG 169. Commissioned by the National Institute for Health and Care Excellence. August 2013; 61-63.

65. Jämsen E, Nevalainen P, Kalliovalkama J, Moilanen T. Preoperative hyperglycemia predicts infected total knee replacement. Eur J Intern Med. 2010 Jun;21(3):196-201. https://doi. org/10.1016/j.ejim.2010.02.006 PMID:20493422

66. Olsen MA, Nepple JJ, Riew KD, Lenke LG, Bridwell KH, Mayfield J, et al. Risk factors for surgical site infection following orthopaedic spinal operations. J Bone Joint Surg Am. 2008 Jan;90(1):62-9. https://doi. org/10.2106/JBJS.F.01515 PMID:18171958

67. Richards JE, Kauffmann RM, Zuckerman SL, Obremskey WT, May AK. Relationship of hyperglycemia and surgical-site infection in orthopaedic surgery. J Bone Joint Surg Am. 2012 Jul;94(13):11816. https://doi.org/10.2106/ JBJS.K.00193 PMID:22760385

68. Feringa $\mathrm{HH}$, Vidakovic $\mathrm{R}, \mathrm{Ka}-$ ragiannis $S E$, Dunkelgrun $M$, Elhendy A, Boersma E, et al. Impaired glucose regulation, elevated glycated haemoglobin and cardiac ischaemic events in vascular surgery patients. Diabet Med. 2008 Mar;25(3):3149. https://doi.org/10.1111/ j.1464-5491.2007.02352.x PMID:18201208

69. McGirt MJ, Woodworth GF, Brooke BS, Coon AL, Jain S, Buck D, et al. Hyperglycemia independently increases the risk 
of perioperative stroke, myocardial infarction, and death after carotid endarterectomy. Neurosurgery. 2006 Jun;58(6):106673. https://doi.org/10.1227/01. NEU.0000215887.59922.36 PMID:16723885

70. Noordzij PG, Boersma E, Schreiner $F$, Kertai MD, Feringa $\mathrm{HH}$, Dunkelgrun $M$, et al. Increased preoperative glucose levels are associated with perioperative mortality in patients undergoing noncardiac, nonvascular surgery. Eur J Endocrinol. 2007 Jan;156(1):137-42. https:// doi.org/10.1530/eje.1.02321 PMID:17218737

71. Dunkelgrun $M$, Schreiner $F$, Schockman DB, Hoeks SE, Feringa HH, Goei D, et al. Usefulness of preoperative oral glucose tolerance testing for perioperative risk stratification in patients scheduled for elective vascular surgery. Am J
Cardiol. 2008 Feb;101(4):5269. https://doi.org/10.1016/j. amjcard.2007.09.103 PMID:18312771

72. van Kuijk JP, Dunkelgrun M, Schreiner F, Flu WJ, Galal W, van Domburg RT, et al. Preoperative oral glucose tolerance testing in vascular surgery patients: long-term cardiovascular outcome. Am Heart J. 2009 May;157(5):919-25. https://doi. org/10.1016/j.ahj.2009.02.011 PMID:19376322

73. Abdelmalak BB, Knittel J, Abdelmalak JB, Dalton JE, Christiansen E, Foss J, et al. Preoperative blood glucose concentrations and postoperative outcomes after elective non-cardiac surgery: an observational study. $\mathrm{Br}$ J Anaesth. 2014 Jan;112(1):7988. https://doi.org/10.1093/bja/ aet297 PMID:24009267

74. Kotagal $M$, Symons RG, Hirsch IB, Umpierrez GE, Dellinger
EP, Farrokhi ET, et al.; SCOAPCERTAIN Collaborative. Perioperative hyperglycemia and risk of adverse events among patients with and without diabetes. Ann Surg. 2015 Jan;261(1):97103. https://doi.org/10.1097/ SLA.0000000000000688 PMID:25133932

75. Chee $Y L$, Crawford JC, Watson HG, Greaves M; British Committee for Standards in Haematology. Guidelines on the assessment of bleeding risk prior to surgery or invasive procedures. $\mathrm{Br} J$ Haematol. 2008 Mar;140(5):496504. https://doi.org/10.1111/ j.1365-2141.2007.06968.x PMID:18275427

76. Drekonja DM, Zarmbinski B, Johnson JR. Preoperative urine cultures at a veterans affairs medical center. JAMA Intern Med. 2013 Jan;173(1):71-2. https:// doi.org/10.1001/2013.jamainternmed.834 PMID:23212291 